\title{
Einleitung: Desistance als konzeptioneller Rahmen für die Untersuchung von Reintegrationsprozessen
}

\author{
Peter Rieker · Jakob Humm • Franz Zahradnik
}

C) Springer Fachmedien Wiesbaden 2016

\section{Desistance - Skizzierung eines Forschungskonzepts}

Mit Desistance ist das „Abstandnehmen“ von kriminellem Verhalten gemeint, d. h. es geht dabei um Prozesse der Beendigung einer kriminellen Karriere. Mit dem Desistance-Konzept grenzt man sich in der kriminologischen Forschung von Perspektiven ab, die unter den Stichworten „,kriminelle Karriere“ und „Rückfall“ vor allem abweichendes Verhalten in den Fokus rücken. Durch diese bewusste Verschiebung der Aufmerksamkeit von der Frage „Wie entsteht abweichendes Verhalten?“ zur Frage „Wie wird abweichendes Verhalten beendet?“ erfahren die späteren Phasen einer kriminellen Karriere erhöhte Aufmerksamkeit. Auf diese Weise trägt man solchen Erkenntnissen Rechnung, denen zufolge das Ausbleiben weiterer Delinquenz nicht einfach durch eine nachlassende Bedeutung oder Wirksamkeit der Bedingungen bzw. Prozesse erklärt werden kann, die für die Entstehung und Verfestigung von Delinquenz verantwortlich gemacht werden. Stattdessen ist auch die Beendigung einer kriminellen Karriere als Prozess anzusehen, der bestimmter Voraussetzungen, Haltungen und Handlungen bedarf. Desistance ist dabei nicht nur mit aktiven Bemühungen, bewussten Entscheidungen und veränderten Handlungsweisen verbunden, sondern bedarf auch bestimmter sozialer Bedingungen, psychischer und sozialer Ressourcen sowie professioneller fachlicher Unterstützung. Es kann als Verdienst der Desistance-Forschung angesehen werden, diese voraussetzungsvollen Prozesse, ihre Bedingungen und Verläufe sichtbar zu machen. Grundsätzlich lässt sich das

P. Rieker $(\bowtie) \cdot$ J. Humm · F. Zahradnik Institut für Erziehungswissenschaft, Universität Zürich, Freiestrasse 36, 8032 Zürich, Schweiz E-Mail: prieker@ife.uzh.ch

J. Humm

E-Mail: jakob.humm@ife.uzh.ch

F. Zahradnik

E-Mail: franz.zahradnik@ife.uzh.ch 
Desistance-Konzept reibungslos in bestehende Karriere-Konzepte einfügen, insofern hier eine prozessorientierte Sichtweise deutlich wird, die das Wechselspiel zwischen denkenden, fühlenden und handelnden Subjekten einerseits und gesellschaftlichen Bedingungen und Reaktionen andererseits in den Blick nimmt. Man könnte sogar sagen, dass traditionelle Modelle krimineller Karrieren (Becker 1981; Quensel 1973; Thornberry 1987) durch Desistance-Konzepte ergänzt und vervollständigt werden.

Forschungen zu Desistance wurden bisher vor allem im anglo-amerikanischen Raum realisiert und können auf eine gewisse Tradition zurückblicken, die bis zu der Studie von Sheldon und Eleanor Glueck (Glueck und Glueck 1968) zurückverfolgt werden kann. Während die kriminologische Rückfallforschung in methodischer Hinsicht eher quantitativ orientiert ist, sind im Kontext der Desistance-Forschung vermehrt qualitative Zugänge festzustellen, was mit der Bedeutung subjektiv-individueller Perspektiven in Zusammenhang stehen dürfte, die im Kontext von Desistance-Prozessen als relevant gelten. Mit der zunehmenden Entwicklung dieses Forschungsgebiets festigten sich unterschiedliche theoretische Modelle, welche sich im Wesentlichen in drei Ausrichtungen bewegen. Etabliert haben sich zum einen die sozio-strukturellen Annahmen, welche davon ausgehen, dass primär äußere Bedingungen dazu führen, dass es zu einem Abbruch des delikthaften Verhaltens kommt. Dem gegenüber stehen Grundüberlegungen, welche davon ausgehen, dass es vor allem individuelle, kognitive Veränderungen sind, welche dazu beitragen, dass eine Abkehr von einem deliktbehafteten Leben ermöglicht wird. Aktuelle Forschungen versuchen, diese beiden Ansätze zu vereinen und beschreiben konsequenterweise das Zusammenspiel zwischen äußeren Chancen und inneren Veränderungsprozessen als die Faktoren, welche einen Desistance-Prozess anzustoßen vermögen. Diese verschiedenen theoretischen Konzepte werden nachfolgend ausführlicher skizziert.

\section{Grundlegende Theorien der Desistance-Forschung}

Robert Sampson und John Laub (1997) betonen in ihrer ,altersabhängigen Theorie informeller sozialer Kontrolle“ die Bedeutung der aktuellen Lebensumstände und der sozialen Bindungen für Desistance-Prozesse (ehemaliger) Delinquenter. Ausgehend von der Kontrolltheorie von Gottfredson und Hirschi (1990) verstehen sie abweichendes Verhalten als das Produkt schwacher Bindungen an die jeweilige Gesellschaft und mangelnder sozialer Kontrolle. Dementsprechend gehen Sampson und Laub davon aus, dass eine Intensivierung sozialer Bindungen, z. B. durch eine Eheschließung oder die Etablierung einer stabilen Arbeitsanstellung, wesentlich dazu beitragen kann, ehemals Delinquente zu einem normenkonformen Leben zu bewegen. Dieses Konzept wurde inzwischen mehrfach empirisch bestätigt (z. B. Stelly und Thomas 2004) und gilt daher als besonders robuste Desistance-Theorie. Kritik am Konzept von Sampson und Laub bezieht sich u. a. darauf, dass die Entstehung und Stärkung stabiler Bindungen sowie der Stellenwert dieser Bindungen für die Legalbewährung nicht klar expliziert wurden (Hofinger 2012).

Maruna (2001) weist im Unterschied zu Sampson und Laub auf die Bedeutung der subjektiven Perspektiven bzw. des Selbstbildes (ehemals) Delinquenter für die Beendigung von Delinquenzkarrieren hin. Aufgrund inhaltsanalytischer Auswertung der 
Interviews mit 65 Ex-Straftätern unterscheidet er zwischen Desistern und Persistern, wobei er sich dabei auf die Selbst-Narrative der Interviewten stützt. Nach Maruna entwerfen die Persister ein ,condemnation script“ (Skript der Verdammung), d. h. sie begründen ihre kriminelle Vergangenheit in vielen Fällen mit einer unglücklichen Kindheit, einer verhängnisvollen Drogenkarriere oder Schicksalsschlägen, wodurch sie ihrer Entwicklung etwas Unausweichliches geben und sich als Opfer stilisieren. Dem gegenüber entwerfen die Desister ein ,redemption script“ (Skript der Erlösung), in welchem sie eine Entwicklung zur Eigenverantwortlichkeit skizzieren, d. h. sie bezeichnen ihre kriminelle Vergangenheit als Chance, der Gesellschaft etwas zurückzugeben bzw. bemühen sich um eine Kompensation der selbst verursachten Schäden - beispielsweise als Mentoren für Jugendliche oder durch die Mitarbeit bei gemeinnützigen Vereinen. Auch Marunas Theorie wurde stark kritisiert (Laub und Sampson 2003), wobei vor allem die Annahme der „Identitätsrekonstituierung“ auf vehementen Widerstand stieß.

Neuere Tendenzen der Desistance-Forschung bemühen sich darum, die oben skizzierten Ansätze zu integrieren, und betonen die Wechselbeziehungen und die kompensatorische Beeinflussung der Faktoren, welche einen Desistance-Prozess beeinflussen können. So gehen Giordano et al. (2002) davon aus, dass das Zusammenspiel von strukturellen Möglichkeiten (soziale Bindungen, Arbeitsplatz u. ä.m.) und der eigenen Handlungsfähigkeit zentral dafür ist, ein deliktfreies Leben zu führen, wobei das Individuum durch eine Veränderung seiner Einstellung selbst den Schlüssel für diese Entwicklung in der Hand hat. Während Giordano et al. das Individuum und seine mögliche Verhaltensänderung ins Zentrum stellen, fokussieren Farrall et al. (2010) primär auf die soziostrukturellen Gegebenheiten. Ihrer Ansicht nach ist zwar die eigene Handlungsfähigkeit von zentraler Bedeutung, diese sei aber immer von strukturellen Rahmenbedingungen innerhalb eines sozialen und historischen Kontextes abhängig.

\section{Implikationen der Desistance-Forschung}

Die Desistance-Forschung untersucht Bedingungen und Prozesse der Reintegration ehemaliger Straftäter und formuliert auf dieser Grundlage auch konkrete Empfehlungen für die Praxis (vgl. Farrall 2012; Halsey und Deegan 2015; Maruna 2001; McNeill et al. 2012). Anregungen werden vor allem in Hinblick auf die Ausgestaltung des eigentlichen Vollzugs, die Unterstützung beim Übergang aus den betreuenden Institutionen sowie die Gestaltung von Hilfebeziehungen formuliert.

Obwohl sich die Desistance-Forschung nicht um die Ausgestaltung konkreter Vollzugsformen bemüht, scheinen gewisse Erkenntnisse nun doch auch vermehrt in die praktische Arbeit der Institutionen des Strafvollzugs einzufließen (McNeill et al. 2012). So zeigt sich, dass in der Vollzugsarbeit inzwischen verstärkt eine individuelle Orientierung als sinnvoll und notwendig erachtet wird, sei es durch Stärkung der subjektiven Ressourcen, sei es durch individuell ausgestaltete Unterstützungsangebote oder durch eine ausgeprägtere Berücksichtigung der Bedürfnisse der Inhaftierten (Dietrich 2011; Müller und Rossi 2009). Zunehmend durchgesetzt hat sich auch die Position, dass durch Interventionen seitens der Institutionen Desi- 
stance-Prozesse lediglich angestoßen und gegebenenfalls unterstützt werden können, dass auf diese Weise aber nicht schon eine Legalbewährung vermittelt werden kann.

Ergebnisse der Desistance-Forschung verweisen auf die Bedeutung geplanter und bewusst gestalteter Übergänge aus betreuenden Institutionen in die Freiheit (Wirth 2014). Dabei gelten ein stabiles soziales Umfeld, die Einbindung in Arbeit (Schinkel 2014, S. 116) sowie eine „empfangende“ Gemeinschaft als wichtige Aspekte. Dementsprechend sollen Entlassungen längerfristig und frühzeitig eingeleitet, Hilfestellungen bei Wohnungs- und Arbeitssuche zur Verfügung gestellt und für die Entlassenen ermöglicht werden, auch nach der Entlassung wieder Kontakt mit den Diensten der Institution aufzunehmen. Auch für die Bewährungshilfe lassen sich aus der Desistance-Forschung bestimmte Empfehlungen ableiten. Maruna (2001) schlägt vor, ehemaligen Straftätern längerfristige Unterstützung zu bieten und dafür auch Gruppenzusammenhänge zu nutzen. Zudem wird es von Maruna als sinnvoll erachtet, im Rahmen der Bewährungshilfe gezielt zu versuchen, konstruktive Sichtweisen zu fördern und die Klienten bei der Entwicklung solcher Selbstbilder zu fördern, die Alternativen zu delinquenzaffinen Identitäten bieten. Schließlich sollten ehemaligen Straftätern auch Möglichkeiten zur Verfügung gestellt werden, sich für das Gemeinwesen zu engagieren (Hofinger 2012).

Aus der Desistance-Forschung werden auch generelle Konsequenzen für die Gestaltung von Hilfebeziehungen und für den Charakter der gewährten Unterstützung abgeleitet. Besondere Bedeutung wird der Gestaltung von Hilfebeziehungen zugerechnet, wobei der Bewährungshilfe nach Rex (1999) vor allem dann ein Beitrag zu positiv erlebten Veränderungen zugerechnet wurde, wenn die Klienten den Eindruck hatten, dass sie diese Beziehung gemäß ihrer eigenen Bedürfnisse gestalten konnten. In Hinblick auf die benötigte Hilfe wird einerseits die Position vertreten, dass es vor allem wichtig ist, (ehemaligen) Straftätern einen Raum für die Äußerung ihrer Erfahrungen und Sichtweisen zur Verfügung zu stellen und ihnen gut zuzuhören (McCulloch 2005), zudem bedürfe es einer wohlwollenden Kommunikationskultur zwischen Betreuten und Fachkräften (Maruna 2001). Andererseits wird die große Bedeutung lebenspraktischer Hilfe betont, z. B. bei der Arbeits- und Wohnungssuche oder beim Umgang mit belasteten sozialen Beziehungen bzw. bei deren Erneuerung (Farrall 2012; Mills und Codd 2008). Kontrovers wird auch diskutiert, ob die von Maruna (2001) geforderte Unterstützung bei der Entwicklung positiver Selbstkonzepte mit der an anderer Stelle geforderten Aufarbeitung der eigenen Straftaten vereinbar ist (Hofinger 2012).

Angesichts solch hoher Erwartungen, die teilweise auch noch mit einem gewissen Enthusiasmus vertreten werden, darf nicht übersehen werden, dass ehemalige Straftäter sich immer wieder mit sozialen Vorurteilen, Stigmatisierungen und mit Ablehnung durch Angehörige der „Normalgesellschaft“ konfrontiert sehen, die ihre Reintegration erschweren (Farrall und Calverley 2006, S. XI; Stehr 2010, S. 15 ff.). Solche Vorbehalte auf Seiten der „Normalgesellschaft“, die für ehemalige Straffällige enorme Hindernisse auf dem Weg zur Reintegration darstellen können, müssen berücksichtigt werden, wenn man die Erfolge von Desistance-Prozessen bilanziert. 


\section{Zum vorliegenden Heft}

Die in den USA und in Großbritannien etablierte Desistance-Forschung wurde im deutschen Sprachraum bereits vereinzelt rezipiert (Hofinger 2012; Stelly und Thomas 2004), doch sind empirische Studien, die sich auf diesen Ansatz beziehen, nach wie vor selten. Im vorliegenden Heft werden Untersuchungen aus Deutschland, Österreich und der Schweiz präsentiert, die sich an Konzepten der DesistanceForschung orientieren. Damit soll ein aktueller Einblick in die deutschsprachige Desistance-Forschung gegeben werden, um dazu beizutragen, diesen Ansatz auch hierzulande stärker der fachlichen Diskussion zugänglich zu machen. Damit wird nicht nur ein Einblick in gegenwärtige Forschungen auf diesem Gebiet gegeben, sondern es wird auch deutlich, welche unterschiedlichen Aspekte von DesistanceProzessen bereits in den Blick genommen werden. Bei einem Großteil der hier vorgestellten Studien handelt es sich um Längsschnittuntersuchungen, die von ihrer Anlage her geeignet sind, der für die Desistance-Forschung geforderten Prozessorientierung gerecht zu werden.

\section{Die einzelnen Beiträge}

Elke Wienhausen-Knezevic beschreibt in ihrem Beitrag die sozialen Passagen jugendlicher Straftäter aus der Gefangenschaft in die Freiheit, wobei sie dem Aspekt der selbst zugeschriebenen und sprachlich artikulierten Handlungsfähigkeit (Agency) ein besonderes Augenmerk widmet. Grundlage der Studie waren 24 Jugendliche mit sehr unterschiedlichen Deliktkarrieren und Hafterfahrungen. Die Daten wurden mittels halbstrukturierter Interviews erhoben und anschließend im Sinne des interpretativen Paradigmas analysiert. Mithilfe des von der Autorin entwickelten ZARIASchemas (Ziele, Agency, Ressourcen, Identiät und Anerkennung) werden anhand von drei Fallbeispielen unterschiedlich verlaufende Reintegrationsprozesse nachgezeichnet und die kompensatorische Wechselwirkung der beeinflussenden Faktoren skizziert. Als besonders herausfordernd für die Reintegration bezeichnet die Autorin die Schwierigkeiten bei der Herstellung und Aufrechterhaltung von sozialen Kontakten sowie die wiederholt erlebte soziale Diskriminierung aufgrund der Inhaftierung. Der Text unterstreicht die These, dass Desistance-Verläufe nicht gradlinige Prozesse sind, sondern sich in den meisten Fällen Zick-Zack-artig gestalten.

Franz Zahradnik und Jakob Humm stellen in ihrem Beitrag Desistance-Prozesse junger Männer in den Mittelpunkt, die eine mehrjährige Maßnahme für junge erwachsene Straftäter in der Schweiz absolviert haben. Anhand zweier Fallinterpretationen, die auf wiederholten Interviews einer Längsschnittstudie basieren, zeigen sie auf, wie Beziehungserfahrungen in unterschiedlichen Bereichen ineinandergreifen. Die je eigensinnigen Deutungs- und Verarbeitungsweisen von institutionellen Interventionen sowie der anschließenden Übergänge stehen in engem Zusammenhang mit den erfahrenen Beziehungsqualitäten zu professionellen und persönlichen Bezugspersonen. Im professionellen Bereich bieten vor allem authentische und vertrauensvolle Unterstützungs- und Beziehungsangebote Anknüpfungspunkte für die Herausbildung und Stabilisierung neuer Selbstentwürfe. Hierbei sind jedoch deutli- 
che Unterschiede hinsichtlich der fallspezifischen Sichtweisen auf den sozialtherapeutischen wie auch berufsbildenden Bereich festzustellen. Die Übergänge weisen ebenso fallbezogene Besonderheiten auf, da sie im Kontext von unterschiedlich gelagerten Konfliktdynamiken vollzogen werden müssen. In Hinblick auf Desistance wird die jeweils spezifische Einbindung in generative Strukturen sichtbar, der von den jungen Männern auch eine Schutzfunktion vor einem erneuten Abgleiten in die Delinquenz zugeschrieben wird.

Steffen Zdun präsentiert in seinem Beitrag Ergebnisse aus einer qualitativen Längsschnittstudie, in deren Rahmen mit 18 männlichen Heranwachsenden wiederholt halbstrukturierte Interviews geführt werden konnten. Der Fokus richtet sich auf die Bedeutung von Freundschaften und Partnerschaften für Desistance-Prozesse. Bei der Fallauswahl wurden Delinquenzverläufe mit unterschiedlicher Deliktschwere berücksichtigt, sodass die Varianz von eher kleinkriminellen Tendenzen bis hin zu ausgeprägten kriminellen Karrieren reicht. Der Einfluss von Partnerinnen wird von den jungen Männern durchweg als prosozial beschrieben, da sie in den Beziehungen Impulse für neue Sichtweisen jenseits von Delinquenz setzen. Je nach subjektiver Bedeutung, die der Partnerin beigemessen wird, können Entwicklungsprozesse in Richtung Desistance angestoßen und unterstützt werden. Teilweise werden schon durch Beziehungen, die nur kurze Zeit andauern, erste Schritte einer anhaltenden Stabilisierung in Gang gesetzt. Ein zentraler Befund besteht darin, dass keine direkte Verbindung zwischen gescheiterten Beziehungen und Rückfällen festgestellt wurde. Bei den Freundschaften wird von den Interviewten eine deutliche Unterscheidung zwischen guten und schlechten Freunden gemacht. $\mathrm{Zu}$ schlechten Freunden werden frühere delinquente Weggefährten erklärt, von denen man sich distanzieren möchte. Gute Freunde sind hingegen entweder alte Bekannte, die eigentlich schon immer helfen wollten, oder aber neue Kontakte, die keinen delinquenten Hintergrund aufweisen. Am Ende des Beitrags wird noch eine spezifische Form der Selbstdeutung skizziert, die mit dem Begriff „,self-made man“ gefasst wird.

Anke Neuber beschäftigt sich in ihrem Beitrag mit Desistance-Prozessen von jungen inhaftierten Frauen. Aus einer adoleszenz- und geschlechtertheoretischen Perspektive stellt sie die grundlegende Frage, mit welchen theoretischen und methodischen Zugangsweisen Desistance-Prozesse angemessen untersucht werden können. Anhand des Fallbeispiels einer jungen Frau, die in einer breiter angelegten qualitativen Längsschnittuntersuchung mehrfach interviewt wurde, arbeitet sie die biographischen Konfliktmuster heraus, die sich in der Adoleszenz mit DesistanceProzessen verbinden können. Hierbei werden die Parallelen zu Integrations- und Autonomiekonflikten von jungen inhaftierten Männern deutlich, womit zugleich geschlechterstereotype Zuschreibungen, wie sie in der Forschung zu abweichendem Verhalten vorherrschen, zurückgewiesen werden. Abschließend plädiert die Autorin für eine integrierte Perspektive, die der Komplexität der Kategorie Geschlecht dahingehend gerecht wird, dass geschlechtsbezogene Unterschiede aufmerksamer in Hinblick auf strukturelle, kulturelle und biographische Einflüsse untersucht werden, indem die unterschiedlichen Dimensionen analytisch nicht vorschnell miteinander verquickt werden.

Veronika Hofinger konzentriert sich in ihrem Beitrag auf die Vereinbarkeit von individuellen Bedürfnissen der Inhaftierten mit einem strukturierten, therapeutischen 
Vorgehen. Diese Untersuchung wurde realisiert, nachdem in Österreich die Bewährungshilfe neu konzipiert und dabei die strukturierte Deliktaufarbeitung verpflichtend implementiert worden war. Auf diese Weise konnten sowohl die Einschätzungen der Inhaftierten in Bezug auf die inzwischen vereinheitlichte Betreuung erhoben, als auch Problemfelder der Profession der Bewährungshelfer erkannt werden. Hinzu kommt die Erkenntnis, dass die bis anhin gültige Trennung zwischen einer ,what works-Perspektive“" und der Einbeziehung subjektiver Perspektiven nicht zwingend notwendig ist. Es zeigt sich, dass die Studienteilnehmer der vereinheitlichten Deliktaufarbeitung mehrheitlich positiv gegenüber stehen. Die Autorin verweist abschließend auf die notwendigen Gelingensbedingungen einer nachhaltigen Verknüpfung von Subjektorientierung und strukturierter Zielformulierung in der Bewährungshilfearbeit.

Danksagung Das vorliegende Schwerpunktheft wäre ohne die tatkräftige Mitarbeit verschiedener Kolleginnen und Kollegen nicht möglich gewesen. Zunächst möchten wir uns bei den Autorinnen und Autoren bedanken, die nicht nur die Beiträge zu diesem Heft beigesteuert haben, sondern auch bereit waren, Anregungen der Reviews und der Herausgeber aufzugreifen und die Texte zeitnah zu überarbeiten. Unser Dank gebührt auch den anonymen Reviewer/inne/n, die ungeachtet ihrer sonstigen Verpflichtungen bereit waren, die Beiträge kritisch zu lesen und konstruktive Rückmeldungen zu formulieren. Die Redaktion der Zeitschrift „Soziale Probleme“ war bereit, sich auf das Vorhaben dieses Schwerpunktheftes einzulassen und hat unser Vorhaben von Beginn an in vielfältiger Weise unterstützt, wofür wir uns herzlich bedanken. Für die redaktionelle Durchsicht und für die gründliche Bearbeitung der Beiträge geht ein großer Dank an Daniel Werner.

\section{Literatur}

Becker, Howard S. 1981. Außenseiter. Zur Soziologie abweichenden Verhaltens. Frankfurt am Main: Fischer.

Dietrich, Kathrin. 2011. Soziale Integration krimineller Jugendlicher durch Vollzug in freien Formen und offene Unterbringung - Deutschland und Schweiz im Vergleich. In Erziehung krimineller Jugendlicher in kriminalpädagogischen Institutionen, Hrsg. Susann Reinheckel, 43-58. Wiesbaden: VS.

Farrall, Stephen. 2012. Rethinking What Works with Offenders. Probation, Social Context and Desistance from Crime. New York: Routledge.

Farrall, Stephen, und Adam Calverley. 2006. Understanding Desistance from Crime. Theoretical Directions in Resettlement and Rehabilitation. Berkshire: Open University Press.

Farrall, Stephen, Anthony Bottoms, und Joanna Shapland. 2010. Social Structures and Desistance from Crime. European Journal of Criminology 7(6):546-570.

Giordano, Peggy C., Stephen A. Cernkovich, und Jennifer L. Rudolph. 2002. Gender, crime, and desistance: toward a theory of cognitive transformation. American Journal of Sociology 107(4):990-1064.

Glueck, Sheldon, und Eleanor Glueck. 1968. Delinquents and Nondelinquents in Perspective. Cambridge: Harvard University Press.

Gottfredson, Michael R., und Travis Hirschi. 1990. A General Theory of Crime. Stanford: Stanford University Press.

Halsey, Mark, und Simone Deegan. 2015. Young Offenders: Crime, Prison and Struggles for Desistance. Basingstoke: Palgrave MacMillan.

Hofinger, Veronika. 2012. „Desistance from Crime“ - eine Literaturstudie. Wien. http://www.irks.at/assets/ irks/Publikationen/Forschungsbericht/Desistance_Literaturbericht.pdf. Zugegriffen: 12.01.2016.

Laub, John, und Robert Sampson. 2003. Shared Beginnings, Divergent Lives. Delinquent Boys to Age 70. Cambridge: Harvard University Press.

Maruna, Shadd. 2001. Making Good: How Ex-Convicts Reform and Rebuild their Lives. Washington: American Psychological Association.

McCulloch, Trish. 2005. Probation, Social Context and Desistance: Retracing the Relationship. Probation Journal 62(1):8-22. 
McNeill, Fergus, Stephen Farrall, Claire Lightowler, und Maruna Shadd. 2012. How and Why People Stop Offending: Discovering Desistance. Glasgow: Institute for Research and Innovation in Social Services.

Mills, Alice, und Helen Codd. 2008. Prisoners' Families and Offender Management: Mobilizing Social Capital. Probation Journal 55(1):9-24.

Müller, Daniel, und David Rossi. 2009. Rückfall nach Massnahmevollzug. Eine Studie zur Rückfälligkeit von jungen Erwachsenen aus den Massnahmezentren Arxhof und Uitikon. http://www.baselland.ch/ fileadmin/baselland/files/docs/jpd/arxhof/rueckfallstudie.pdf. Zugegriffen: 12.01.2016.

Quensel, Stefan. 1973. Wie wird man kriminell? In Offensive Sozialpädagogik, Hrsg. Hermann Giesecke, 45-55. Göttingen: Vandenhoeck \& Ruprecht.

Rex, Sue. 1999. Desistance from Offending. Experiences of Probation. The Howard Journal of Criminal Justice 38(4):366-283.

Sampson, Robert J., und John H. Laub. 1997. Life-Course Theory of Cumulative Disadvantages and the Stability of Delinquency. In Developmental theories of crime and delinquency, Hrsg. Terence P. Thornberry, 133-161. New Brunswick London: Transaction Publishers.

Schinkel, Marguerite. 2014. Being Imprisoned: Punishment, Adaptation and Desistance. Basingstoke: Palgrave MacMillan.

Stehr, Johannes. 2010. Gewaltige Angst vor der Jugend ... warum, von wem und auf welche Weise sie geschürt wird. Sozial Extra 34(9):15-18.

Stelly, Wolfgang, und Jürgen Thomas. 2004. Wege aus schwerer Jugendkriminalität. Eine qualitative Studie zu Hintergründen und Bedingungen einer erfolgreichen Reintegration von mehrfachauffälligen Jungtätern. Tübingen: TüKrim.

Thornberry, Terence P. 1987. Towards an Interactional Theory of Delinquency. Criminology 25(4):863-891.

Wirth, Wolfgang. 2014. Übergänge aus dem Jugendstrafvollzug. Brüchige Biografien - Abzubrechende „Nacherziehung“ - Ununterbrochenes Übergangsmanagement, Referat. Wissenschaftliche DJI-Jahrestagung „Risikokarrieren im Kindes- und Jugendalter“, Berlin, 11. und 12. November 2014. http://www.dji.de/fileadmin/user_upload/dasdji/news/2014/2014-JT/F2_02_Wirth_DJI_ Jahrestagung_2014.pdf. 